\title{
nature
}

\section{Business and pleasure can mix}

The British government should not miss an opportunity to show its support for an innovative scheme from the Wellcome Trust to help take genomics research from the lab to the market.

n the next few months, Britain's deputy prime minister and environment secretary, John Prescott, will face a particularly tricky decision. He has to decide, on the basis of evidence presented last week at a public inquiry, whether to allow the Wellcome Trust, the world's largest medical research charity, to extend its genome-research campus at Hinxton, a rural location ten miles south of Cambridge, to provide 'incubator' space for spin-off companies (see pages 355-356).

The trust is not planning an ordinary research park. It wants to create an environment where the boundaries between fundamental research and its commercial applications - and between work and leisure - are blurred. It has a vision of ground-breaking ideas in genomics-based health care emerging from both planned and chance interactions between scientists and entrepreneurs.

The proposed extension includes shared conference rooms and coffee bars, a cricket field, and facilities for bird-watching, fishing and other leisure pursuits. Equally important, potential entrepreneurs will have access to purpose-built accommodation on terms the market will find hard to match. But plans for the extension have been rejected by the local council on the grounds that any new development on agricultural land away from public transport contravenes government policies to protect the countryside and discourage journeys by car.

The trust has tried to meet the content, if not the spirit, of such objections. It has promised to put in place measures to meet most of the local authority's targets to reduce the number of car journeys. It has also promised to pay for extra bus services and to penalize cars parked on the extension without authorization. And it insists that there will be no commercial manufacturing on the site, and that companies that survive more than six years will be asked to move elsewhere.

Prescott, therefore, has fewer reasons than initially to refuse the trust's application. He may be tempted to do so from Wellcome's lessthan-convincing defence of its claim that companies housed within walking distance of research laboratories perform better than those further away. This will be a tricky issue for the deputy prime minister, as there is little empirical evidence to support the case for 'co-location'. Supportive words from those who have already enjoyed such arrangements are not the same as hard data on its relative effectiveness.

But an absence of hard numbers should not be a reason to reject what is an innovative - and, in its revised form, a much more 'environmentally friendly' - scheme, with the potential to contribute significantly to our treatment of disease. The trust has shown it is willing to accommodate local concerns, even if it needs to listen more and work harder to allay negative perceptions of itself. It is time for South Cambridgeshire District Council and local residents to join in the spirit of compromise and lift their objections.

\section{Pulling together in Latin America}

\section{Greater pan-regional scientific collaboration could raise Latin American science to the next level of excellence.}

atin America, whatever the recent economic travails of Brazil, is well placed to strengthen its already growing contribution to the world of science (as a special supplement to this issue demonstrates). Admittedly, day-to-day circumstances remain difficult for scientists in the region. Progress sometimes seems to be faltering, with governments continually introducing new schemes to support science - and then failing to find the money to follow them through.

But the international influence of Latin American scientists is steadily expanding, and there is every indication that it will continue to do so. Certainly, the overall trajectory, measured, for want of a better yardstick, by the proportion of papers from the region published in international journals, points firmly upwards.

As scientists from the region's widely disparate countries jostle to both compete and collaborate with their better-supported peers in the United States and Europe, however, it is becoming increasingly important for them to recognize how much they can learn from the experience of their neighbours. Most would, indeed, like to work more closely with their peers in neighbouring countries. At present, though, there is little encouragement to do so.

Most of these researchers trained in the United States or in Europe, and are inevitably keen to maintain strong connections with the wealthy research institutions of the so-called developed world. It is certainly far easier to work within an international collaboration if at least one end of that collaboration is adequately funded.

There are plenty of isolated examples of scientific collaboration between the countries of the region. At present, however, there is no overall strategy to pool resources in a way that would strengthen the region's scientific base. Many officials - and some scientists - continue to believe that the only worthwhile activity in their particular area of interest lies far to the north.

But research collaboration between north and south is inevitably asymmetrical, with limits on its usefulness to the weaker party. As Latin American science grows in scope and confidence, the region's relatively small scientific communities will greatly increase their ability to compete internationally by combining forces to create the critical mass needed to produce high-quality science in their own region.

In Europe, scientific collaboration has played a significant role in boosting national scientific capabilities, particularly when faced with competition from the United States. The European Laboratory for Particle Physics (CERN) and the European Molecular Biology Laboratory are just two examples of what has been achieved. Latin America now faces a similar challenge.

A number of proposals under consideration, such as the IberoAmerican Molecular Biology Organization's plans for a centre of excellence and the World Bank's Millennium Institutes, could catalyse a regional response. President Eduardo Frei of Chile has tried apparently with little success - to place research collaboration on the agenda at summits of the region's leaders. If they want to build competitive economies, the region's main powers - Brazil, Argentina and Mexico - must address the need for pan-regional collaboration. 